\title{
Enhanced Production of Bacterial Cellulose in Komagataeibacter xylinus Via Tuning of Biosynthesis Genes with Synthetic RBS
}

\author{
Dong Hoon Hur ${ }^{1}$, Woo Sung Choi ${ }^{1}$, Tae Yong Kim ${ }^{2}$, Sang Yup Lee ${ }^{1}$, Jin Hwan Park ${ }^{2 *}$, and \\ Ki Jun Jeong ${ }^{1,3 *}$ \\ ${ }^{1}$ Department of Chemical and Biomolecular Engineering, BK21 Plus Program, Korea Advanced Institute of Science \\ and Technology (KAIST), Daejeon 34141, Republic of Korea \\ ${ }^{2}$ Biomaterials Lab, Samsung Advanced Institute of Technology (SAIT), Samsung Electronics Co., Ltd., Suwon 16678, \\ Republic of Korea \\ ${ }^{3}$ KAIST Institute for the BioCentury, Korea Advanced Institute of Science and Technology (KAIST), Daejeon 34141, \\ Republic of Korea
}

\begin{abstract}
Bacterial cellulose (BC) has outstanding physical and chemical properties, including high crystallinity, moisture retention, and tensile strength. Currently, the major producer of $B C$ is Komagataeibacter xylinus. However, due to limited tools of expression, this host is difficult to engineer metabolically to improve BC productivity. In this study, a regulated expression system for $K$. xylinus with synthetic ribosome binding site (RBS) was developed and used to engineer a $B C$ biosynthesis pathway. A synthetic RBS library was constructed using green fluorescent protein (GFP) as a reporter, and three synthetic RBSs (R4, R15, and R6) with different strengths were successfully isolated by fluorescence-activated cell sorting (FACS). Using synthetic RBS, we optimized the expression of three homologous genes responsible for BC production, pgm, galU, and $n d p$, and thereby greatly increased it under both static and shaking culture conditions. The final titer of BC under static and shaking conditions was 5.28 and $3.67 \mathrm{~g} / \mathrm{l}$, respectively. Our findings demonstrate that reinforced metabolic flux towards $B C$ through quantitative gene expression represents a practical strategy for the improvement of $\mathrm{BC}$ productivity.
\end{abstract}

Keywords: Bacterial cellulose, Komagataeibacter xylinus, synthetic RBS, fluorescence-activated cell sorting

Received: June 18, 2020 Accepted: July 2, 2020

First published online: July 6,2020

${ }^{*}$ Corresponding authors J.H.P.

E-mail:jh111.park@samsung.com K.J.J.

Phone: $+82-42-350-3934$ Fax: +82-42-350-3910 E-mail: kjjeong@kaist.ac.kr;

Supplementary data for this paper are available on-line only at http://jmb.or.kr.

pISSN 1017-7825 elSSN 1738-8872

Copyright (C) 2020 by The Korean Society for Microbiology and Biotechnology

\section{Introduction}

Cellulose is the most abundant biopolymer in the world and is easily found in plants. In addition, various microorganisms, including bacteria, algae, and fungi, produce cellulose. Among bacteria, Komagataeibacter xylinus (previously known as Gluconacetobacter xylinus or Acetobacter xylinum) is a primary producer of bacterial cellulose (BC) [1,2]. BC has outstanding properties, such as high water retention value, surface area, crystallinity, biodegradability, biocompatibility, and high purity due to the absence of lignin and hemicellulose [3-6]. Due to these properties, $\mathrm{BC}$ has potential applications in the biomedical, cosmetic, automotive, packaging, health food, electrical, and sensor industries, with a rapidly increasing commercial value [7-9].

As industrial demand for $\mathrm{BC}$ increases, many efforts have been made to improve its productivity. Most of the research has focused on the optimization of bioprocesses, including culture conditions and medium compositions, among others [10]. However, due to a limited understanding of the $\mathrm{BC}$ biosynthesis mechanism and a lack of efficient genetic tools, there have been few attempts to engineer host organisms for enhanced BC production $[2,8,9]$. Using glucose as a main carbon source of cellulose, $K$. xylinus synthesizes BC by expressing glucokinase $(g k)$, phosphoglucomutase $(p g m)$, UTP-glucose-1-phosphate uridylyltransferase (galU), and bacterial cellulose synthase ( $b c s A B C D$ ) (Fig. 1). The expression of the bcs operon ( $b c s A B C D)$ can be used to increase the production of BC. Mangayil et al. reported that the overexpression of bcs ABCD in K. xylinus DSM 2325 resulted in a production of $\mathrm{BC}$ as high as $4.3 \mathrm{~g} / \mathrm{l}$, which represents a 10.75 -fold increase of $\mathrm{BC}$ produced by the wild-type strain [2]. In addition, recent advances in genome-scale metabolic modeling of BC-producing hosts have highlighted the re-design of metabolic pathways to reinforce the metabolic flux toward BC biosynthesis [11]. This systematic engineering can be further improved by fine-tuning gene expression in several critical steps, as the highest productivity is not achieved under the highest level of gene expression but rather under optimal levels. Recently, two research groups reported on the development of genetic toolkits, including synthetic promoters, RBSs, and plasmids useful for tuning the expression of target genes in Komagataeibacter spp. [12, 13]. Through the 


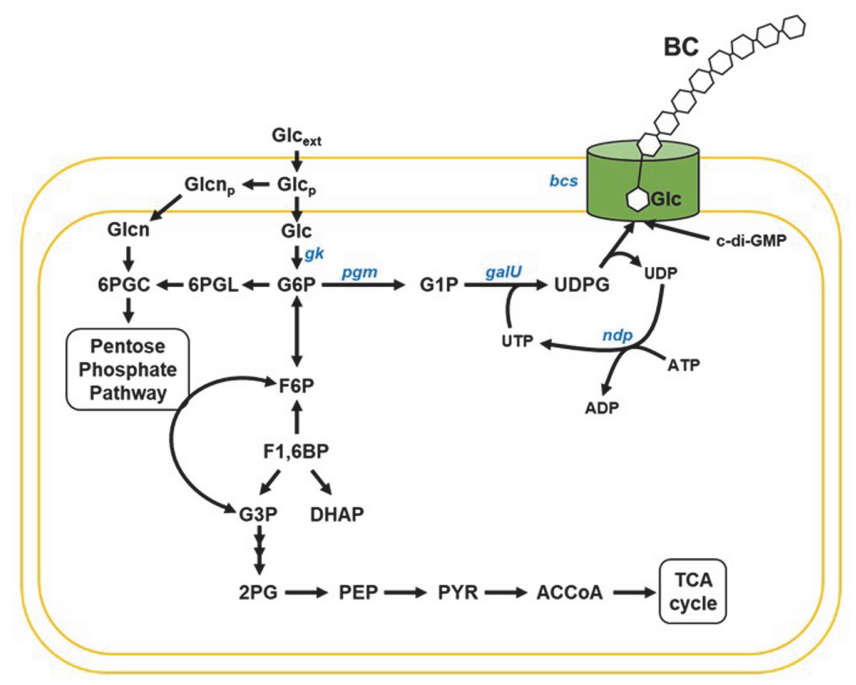

Fig. 1. Metabolic pathway of K. xylinus DSM 2325 and the BC biosynthesis pathway. Metabolite abbreviations are: $\mathrm{Glc}_{\text {exp }}$ D-glucose at extracellular medium; Glc , D-glucose at periplasm; Glc, D-glucose; G6P, D-glucose-6-phosphate; G1P, D-glucose-1-phosphate; UDPG, UDP-glucose; c-di-GMP, cyclic diguanylic acid; $\mathrm{Glcn}_{\mathrm{p}}$, D-gluconate at periplasm; Glcn, Dgluconate; 6PGC, 6-phospho-D-gluconate; 6PGL, 6-phospho-D-glucono-1,5-lactone; F6P, D-fructose-phosphate; F1,6BP, Dfructose-1,6-bisphosphate; DHAP, dihydroxyacetone phosphate; G3P, D-glyceraldehyde-3-phosphoate; 2PG, 2-phospho-Dglycerate; PEP, phosphoenolpyruvate; PYR, pyruvate; ACCoA, acetyl-CoA.

combinatorial assembly of these synthetic toolkits, the production of BC can be increased further, and novel cellulose-based materials can be created. For the reliable and precise control of gene expression, more synthetic parts need to be developed [14-16]. However, the development of fully-synthetic toolkits for the metabolic engineering of Komagataeibacter spp. via high-throughput screening covering numerous sizes of libraries, has not yet been reported.

In this study, we engineered BC-producing K. xylinus using synthetic RBSs capable of tunable gene expression. We constructed a random library of RBSs using GFP as a reporter gene, and three potential RBSs of various strengths were isolated using FACS-based high-throughput screening. The usefulness of the synthetic RBSs was verified, and they were then employed to enhance BC productivity by reinforcing metabolic flux towards $\mathrm{BC}$ biosynthesis.

\section{Materials and Methods}

\section{Bacterial Strains, Media, and Culture Conditions}

All of the bacterial strains used in this study are listed in Table 1. Escherichia coli XL1-Blue was used for cloning and library construction. E. coli were cultivated in Luria-Bertani (LB) medium (10 g/l tryptone, $5 \mathrm{~g} / \mathrm{l}$ yeast extract, $10 \mathrm{~g} / \mathrm{l} \mathrm{NaCl})$ and chloramphenicol $(\mathrm{Cm}, 35 \mathrm{mg} / \mathrm{l})$ was supplemented as a sole antibiotic. K. xylinus DSM 2325 was purchased from the DSMZ (German Collection of Microorganisms and Cell Cultures) and used for RBS screening and BC production. K. xylinus was cultivated in Hestrin-Schramm (HS) medium (50 g/l glucose, $5 \mathrm{~g} / \mathrm{l}$ peptone, $5 \mathrm{~g} / \mathrm{l}$ yeast extract, $2.7 \mathrm{~g} / \mathrm{l}$ anhydrous disodium phosphate, and $1.15 \mathrm{~g} / \mathrm{l}$ citric acid monohydrate) $[17,18]$. For the RBS library screening, cells were cultivated in HS-Cel medium containing $0.4 \%(\mathrm{v} / \mathrm{v})$ cellulase from Trichoderma reesei (Sigma-Aldrich Co., USA). For BC production, $1 \%$ (v/v) ethanol was added to the HS medium. Cells were incubated at $30^{\circ} \mathrm{C}$ for up to 5 days in a static incubator or under shaking $(230 \mathrm{rpm})$ conditions in either $1-2 \mathrm{ml}$ medium in a $14 \mathrm{ml}$ round-bottom culture tube, $25 \mathrm{ml}$ medium in a $125 \mathrm{ml}$ flask for shaking culture or $25 \mathrm{ml}$ medium in 90 (d) $\times 15$ (h) mm Petri dish for static culture. For precise comparison of BC productivity, initial $\mathrm{OD}_{600}$ values were set to 0.02 for all BC production experiments. Seed cultures were carried out in HS-Cel media, and washed with fresh HS medium before transferring to main culture. BC production was carried out for $120 \mathrm{~h}$ in fresh HS medium containing $1 \%(\mathrm{v} / \mathrm{v})$ ethanol. In all cultivation of recombinant $K$. xylinus, $140 \mathrm{mg} / \mathrm{l}$ of chloramphenicol was added as a sole antibiotic.

\section{Plasmid Manipulation and Construction of Synthetic RBS Library in K. xylinus}

All plasmids used in this study are listed in Table 1. The polymerase chain reaction (PCR) was carried out using a C1000 Thermal Cycler (Bio-Rad, USA) and PrimeSTAR HS Polymerase (TAKARA BIO, Inc., Japan). Primer nucleotide sequences used for PCR are listed in Table S1. As a positive control for the RBS screening, we constructed a GFP expression system (pDHJC_sfGFP) by replacing mRFP1 gene with sfGFP and rrnBT1 terminator with a strong terminator, ECK120033736 [13], in J23104-mRFP1-331Bb [12]. For construction of the RBS library, a DNA fragment including a fully randomized RBS sequence (12 bp) and sfGFP was amplified by PCR with primers Fw_Lib and Rv_sfGFP. The PCR product was digested with SpeI and XhoI restriction enzymes, and cloned into the same restriction enzyme sites of pDHJC_sfGFP. The ligated plasmids were transformed with 
Table 1. Bacterial strains and plasmids used in this study.

\begin{tabular}{|c|c|c|}
\hline & Characteristics & Ref. or source \\
\hline \multicolumn{3}{|l|}{ Strains } \\
\hline XL1-Blue & $\begin{array}{l}\text { recA1 endA1 gyrA96 thi-1 hsdR17 supE44 relA1 lac } \\
{\left[F^{\prime} \text { proAB lacI }{ }^{9} Z \mathrm{ZD} 15 \mathrm{Tn} 10\left(\mathrm{Tet}^{\prime}\right)\right]}\end{array}$ & Stratagene $\mathrm{a}^{\mathrm{a}}$ \\
\hline K. xylinus & Wild type & DSM 2325 \\
\hline \multicolumn{3}{|l|}{ Plasmids } \\
\hline J23104-mRFP1-331Bb & pSEVA331 derivative; $\mathrm{P}_{\mathrm{J} 23104}$, Control RBS & [12] Addgene A $^{\mathrm{b}}$ \\
\hline pDHJC_sfGFP & J23104-mRFP1-331Bb derivative; ECK120033736 terminator, sfGFP & This study \\
\hline pDHJC_sfGFP_RBS_lib & pDHJC_sfGFP derivative; sfGFP, RBS library & This study \\
\hline pDHJCR4_sfGFP & pDHJC_sfGFP derivative; sfGFP, RBS R4 & This study \\
\hline pDHJCR15_sfGFP & pDHJC_sfGFP derivative; sfGFP, RBS R15 & This study \\
\hline pDHJCR6_sfGFP & pDHJC_sfGFP derivative; sfGFP, RBS R6 & This study \\
\hline pDHJCR4_pgm & pDHJC_sfGFP derivative; $p g m$, RBS R4 & This study \\
\hline pDHJCR4_galU & pDHJC_sfGFP derivative; galU, RBS R4 & This study \\
\hline pDHJCR4_ndp & pDHJC_sfGFP derivative; $n d p$, RBS R15 & This study \\
\hline pDHJCR15_pgm & pDHJC_sfGFP derivative; $p g m$, RBS R15 & This study \\
\hline pDHJCR15_galU & pDHJC_sfGFP derivative; galU, RBS R15 & This study \\
\hline pDHJCR15_ndp & pDHJC_sfGFP derivative; $n d p$, RBS R15 & This study \\
\hline pDHJCR6_pgm & pDHJC_sfGFP derivative; $p g m$, RBS R6 & This study \\
\hline pDHJCR6_galU & pDHJC_sfGFP derivative; galU, RBS R6 & This study \\
\hline pDHJCR6_ndp & pDHJC_sfGFP derivative; $n d p$, RBS R6 & This study \\
\hline pDHJCR15_pgm_galU_ndp & pDHJC_sfGFP derivative; pgm, galU, $n d p$, RBS R15 & This study \\
\hline
\end{tabular}

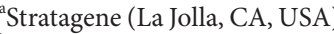

${ }^{\mathrm{b}}$ Addgene (Watertown, MA, USA)

E. coli XL1-Blue by electroporation. The synthetic RBS library in E. coli was purified with Hybrid-Q Plasmid Rapidprep (Geneall, Korea) and then retransformed into K. xylinus by electroporation. Transformed K. xylinus cells were recovered in HS-Cel media containing cellulase for $6 \mathrm{~h}$ and spread on HS agar plates containing $140 \mathrm{mg} / \mathrm{l}$ of chloramphenicol at $30^{\circ} \mathrm{C}$ for $48 \mathrm{~h}$. All of the grown recombinant $K$. xylinus colonies were collected using $300 \mathrm{ml}$ of HS-Cel media and cultured at $30^{\circ} \mathrm{C}$ and $230 \mathrm{rpm}$ to degrade cellulose and make the library homogenous. After $1 \mathrm{~h}$, the recombinant $K$. xylinus cells were stored at $-80^{\circ} \mathrm{C}$ as $15 \%$ glycerol stocks. For the expression of pgm, galU, and $n d p$ genes with three isolated RBSs, each gene was amplified from chromosomal DNA of K. xylinus by PCR with the primer sets listed in Table S1. After the amplification of pgm, galU, and $n d p$, each PCR product was digested with BbsI and BsaI restriction enzymes, and cloned into the NdeI and XhoI sites of pDHJCR4_sfGFP, pDHJCR6_sfGFP and pDHJCR15_sfGFP, yielding pDHJCR4_pgm, pDJHCR4_galU, pDHJCR4_ndp, pDHJCR6_pgm, pDHJCR6_galU, pDHJCR6_ndp, pDHJCR15_pgm, pDHJCR15_galU and pDHJCR15_ndp. For the expression of all pgm, galU and $n d p$ genes in a single plasmid with R15 RBS, vector backbone was amplified using primers Fw_GibV_pDHJCR15 and Rv_GibV_pDHJCR15 using pDHJCR15_sfGFP as a template. A pgm gene was amplified using primers Fw_Gib1_R15_pgm and Rv_Gib1_pgm using pDHJCR15_pgm as a template, and a galU gene was amplified using primers Fw_Gib2_R15_galU and Rv_Gib2_galU using pDHJCR15_galU as a template. An $n d p$ gene was amplified using primers Fw_Gib3_R15_ndp and Rv_Gib3_ndp using pDHJCR15_ndp as a template. All PCR products were mixed and ligated to prepare pDHJCR15_pgm_galU_ndp using Gibson Assembly Master Mix (New England Biolabs, Ipswich, MA, USA) following the manufacturer's manual.

\section{Library Screening Using FACS}

The synthetic RBS library of $K$. xylinus was cultivated in $\mathrm{HS}$-Cel media at $30^{\circ} \mathrm{C}$ for $48 \mathrm{~h}$ with shaking $(230 \mathrm{rpm})$. The fully grown cells were then transferred into fresh HS-Cel media at a final concentration of $1 \%(\mathrm{v} / \mathrm{v})$, and further cultured at $30^{\circ} \mathrm{C}$ and $230 \mathrm{rpm}$ for $16 \mathrm{~h}$. The cells were then screened using FACS (MoFlo XDP; Beckman Coulter, Inc., USA) based on fluorescence intensity detection with a $488 \mathrm{~nm}$ laser and a 530/40 band-pass filter for the sfGFP emission spectrum. The cells with the greatest fluorescence intensity (top 1\%) were sorted and directly inoculated to fresh HS-Cel media, followed by culturing for $48 \mathrm{~h}$. Then, the enriched microbial cultures were transferred into fresh HS-Cel media for the following round of FACS sorting. Starting from the 5th round of sorting, individual clones were randomly selected and inoculated into HS-Cel media for individual analysis of fluorescence intensity. After $48 \mathrm{~h}$ of cultivation, pre-cultured cells were transferred into the main culture and cultivated for $16 \mathrm{~h}$. The fluorescence intensity of each clone was analyzed using FACS.

During the $1^{\text {st }}$ to $3^{\text {rd }}$ rounds of sorting, the 'purify' mode was used to isolate only single droplets containing only fluorescent cells without any negative cells [14]. Using the purify mode, the quality of cell sorting could be highly increased, although with a large fraction of loss. Thus, during the $1^{\text {st }}$ round of sorting, more than $1,000,000$ cells were sorted, while 500,000 cells were sorted during the $2^{\text {nd }}$ and $3^{\text {rd }}$ rounds. During the $4^{\text {th }}$ and $5^{\text {th }}$ rounds, the single cell' mode was used to isolate only single cells separately from cell consortia. Although the loss was even higher than with the purify mode, 50,000 cells were considered to be enough for screening because the cells went through several sorting steps while the library size was $3 \times 10^{7}$. 
A

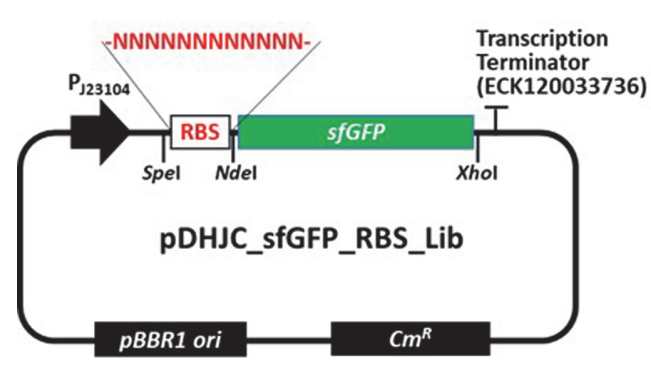

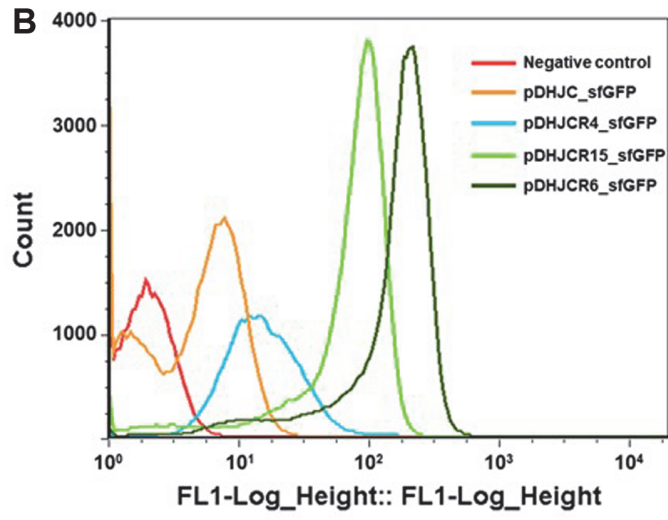

Fig. 2. (A) Plasmid map of pDHJC_sfGFP_RBS_lib and (B) FACS analysis of isolated RBS clones. Totally 50,000 cells were counted for the analysis of negative control and pDHJC_sfGFP, and 100,000 cells were counted for the analysis of pDHJCR4_sfGFP, pDHJCR15_sfGFP and pDHJCR6_sfGFP.

\section{BC Washing and Quantification}

$\mathrm{BC}$ production was measured after several washing procedures to remove any impurities, including cell debris and salts, as described previously with some modifications [19]. The BC was washed twice with distilled water, once with $0.1 \mathrm{M} \mathrm{NaOH}$ solution, and once with distilled water. Each step was carried out using $125 \mathrm{ml}$ of solution, with overnight incubation at $90^{\circ} \mathrm{C}$. After washing, the $\mathrm{BC}$ was dried in an oven at $105^{\circ} \mathrm{C}$ for one week until completely dry. The dehydrated $\mathrm{BC}$ was quantified using a precision scale.

\section{Results and Discussion}

Synthetic RBS Library Construction and Screening

For the construction of the RBS library, a 12 bp RBS region was designed with fully random nucleotide sequences (Fig. 2A). The synthetic RBS library was first constructed in E. coli XL1-Blue, and $3 \times 10^{7}$ colonies were obtained. After the recovery of the library plasmids, these were re-transformed into K. xylinus, for which $2.5 \times 10^{8}$ colonies were obtained. After the cultivation of K. xylinus cells containing the RBS library, the cells with the high fluorescence intensity (FI) (top 1\%) were sorted. In every round, the collected cells were inoculated into fresh HSCel media and cultured for the next round of sorting. During FACS screening, the increase of fluorescence intensity was clearly observed in every round (Fig. S1), which indicated the successful enrichment of cells with RBSs allowing for higher levels of gene expression. After the fifth round of sorting, the collected cells were cultured on HS-Cm agar plates for individual comparison. A total of 40 colonies were randomly picked and the FI in each cell was analyzed using FACS (Fig. S2). Out of 40 clones, the RBS sequences in 20 clones showing different FIs were analyzed. Among them, 10 clones with high levels of FI (mean value, $M=548$ ) were found to contain the same sequence (5'-TACACCGGAGAA-3') (Table 2). An additional 7 clones with medium levels of FI $(M=215)$ contained the same sequence (5'-TAATGAGAGGCC-3'), while three clones with low levels of FI $(M=10)$ had the same sequence (5'-TTACAAAAATGA-30). As a result, three different RBSs were obtained, and named after their representative clone number: R6 (strong FI), R15 (medium FI), and R4 (weak FI) (Fig. 2B). Compared to the control RBS $(M=26)$ that was previously reported by Florea et al. [12], R4 exhibited slightly lower levels of FI, however, the FI levels of R6 and R15 were much higher (21- and 8.3-fold increase, respectively).

\section{Characterization of Isolated Synthetic RBSs}

In gene expression, the translation rate is highly dependent on the thermodynamic structure of the 5 , untranslated region (5' UTR), including the RBS sequence. Based on this thermodynamic analysis, the strength of the isolated RBS can be predicted [20,21]. To understand the strength of the three isolated RBSs, we performed thermodynamic analysis of each RBS using an untranslated region (UTR) designer [20]: 60 base mRNA sequences, including $25 \mathrm{bp}$ of each 5' UTR sequence and the first 35 bases of the sfGFP gene, were used for analysis. As a result, RBS R6 showed the most negative $\mathrm{dG}_{\mathrm{UTR}}$ value, indicating a higher chance of ribosome binding and translation, while $\mathrm{R} 15$ showed a moderate $\mathrm{dG}_{\mathrm{UTR}}$ value, and $\mathrm{R} 4$ showed a positive $\mathrm{dG}_{\mathrm{UTR}}$ value, which correlated to the predicted expression levels (Fig. S3). The results of the thermodynamic analysis indicate an increasing trend in terms of strength in our RBS set.

Table 2. Nucleotide sequences of isolated RBS and corresponding GFP fluorescence intensities.

\begin{tabular}{llc}
\hline RBS & Sequence $\left(5^{\prime}\right.$ to $\left.3^{\prime}\right)$ & Mean fluorescence intensity $(M)$ \\
\hline R6 & TACACCGGAGAA & 548 \\
R15 & TAATGAGAGGCC & 215 \\
R4 & TTACAAAAATGAT & 10 \\
Control $^{\text {a }}$ & GAAAGAGGAGAAA & 26 \\
\hline
\end{tabular}

${ }^{\mathrm{a}} \mathrm{ref} 12$ 
A

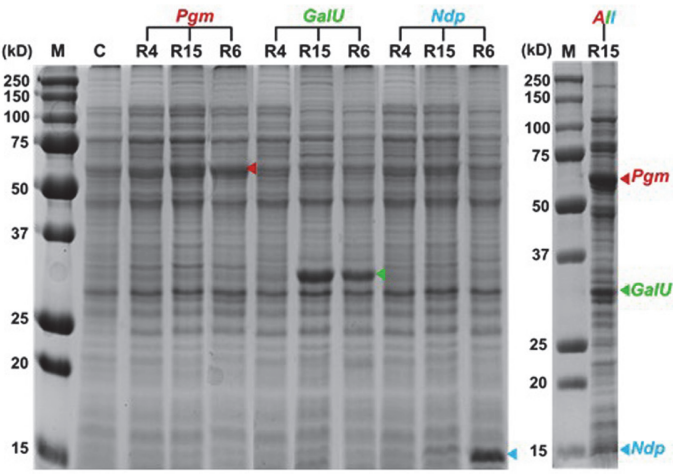

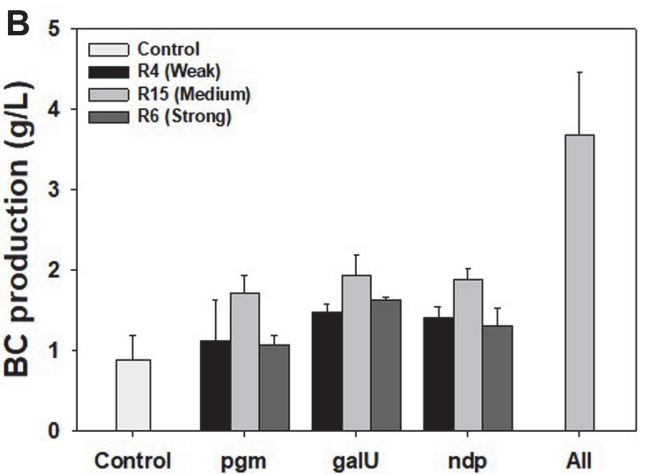

Fig. 3. (A) SDS-PAGE analysis of wild type and recombinant $K$. xylinus expressing either $p g m$, galU, or $n d p$ with each synthetic RBS (R4, R15 or R6) or expressing all three genes with R15 synthetic RBS (right). A wildtype $K$. xylinus without plasmid was used as a control (lane $\mathrm{C}$ ), and $K$. xylinus harboring pDHJCR15_pgm_galU_ndp was used for expression of all genes. Red, green and blue arrowheads represent the band of Pgm, GalU, and Ndp, respectively. (B) BC productivity in recombinant K. xylinus after $120 \mathrm{~h}$ under shaking condition. Control represents a wild-type $K$. xylinus without plasmid.

Expression of BC Synthesis Pathway Genes Using Synthetic RBSs and BC Productivity Under Shaking Condition

Next, to enhance BC production in K. xylinus, the expression levels of the pgm, gal $U$, and $n d p$ genes, which are responsible for the production of $\mathrm{BC}$, were examined using the newly isolated RBSs (R6, R15, and R4). The expression system of each gene was constructed with three different RBSs (a total of 9 sets). After cultivation in liquid HS medium, gene expression in each expression set was analyzed using SDS-PAGE. Among the three genes, the expression of the $n d p$ gene exhibited high expression levels with strong R6 RBS, while pgm and galU exhibited higher expression levels with medium strength R15 (Fig. 3A). In each cultivation, the levels of BC production were also analyzed and compared. Interestingly, among the three RBSs, the use of medium strength R15 resulted in the highest production of BC: BC productivity expressing pgm, gal $U$, and $n d p$ under R15 was 1.71, 1.94, and $1.88 \mathrm{~g} / \mathrm{l}$, respectively, which represents a 1.9-2.2-fold increase compared to the K. xylinus wild type (Fig. 3B). In the case of $n d p$ gene expression under strong R6 RBS, we found that most Ndp was produced in the form of insoluble aggregates (data not shown), which resulted in a lower production of BC (Fig. 3B).

Based on these results, we decided to use RBS R15 for the expression of all three genes, and constructed pDHJCR15_pgm_galU_ndp in which all three genes (pgm, galU, and $n d p)$ were expressed together under $\mathrm{P}_{\mathrm{J} 23104}$ promoter and RBS R15. During the cultivation of K. xylinus harboring pDHJCR15_pgm_galU_ndp, all three genes were found to be highly expressed with high solubilities (Fig. 3A). In addition, the BC production titer was also remarkably increased up to $3.67 \mathrm{~g} / \mathrm{l}$, which was 4.15 -fold higher than the wild-type K. xylinus (Fig. 3B).

BC Productivity Under Static Condition

Finally, to obtain film-like BC, static cultivation was conducted. During the cultivation of the engineered K. xylinus strain, the $\mathrm{BC}$ titer increased rapidly, reaching up to $4.87 \mathrm{~g} / \mathrm{l}$ at $72 \mathrm{~h}$. During further cultivation $(72 \mathrm{~h}$ to $120 \mathrm{~h}$ ), the BC titer increased gradually up to $5.28 \mathrm{~g} / \mathrm{l}$ (Fig. 4). Stagnant production of BC after $72 \mathrm{~h}$ may be caused by lowered mass transfer of $\mathrm{O}_{2}$, depletion of nutrients, cell death, $\mathrm{pH}$ drop, etc. [2]. During the cultivation of the control K. xylinus strain (wild type), the BC titer increased relatively slowly, reaching $4.62 \mathrm{~g} / \mathrm{l}$ after $96 \mathrm{~h}$. The maximum instantaneous BC productivity of the engineered strain was $67.6 \mathrm{mg} / \mathrm{l} / \mathrm{h}$ at $72 \mathrm{~h}$, while that of control

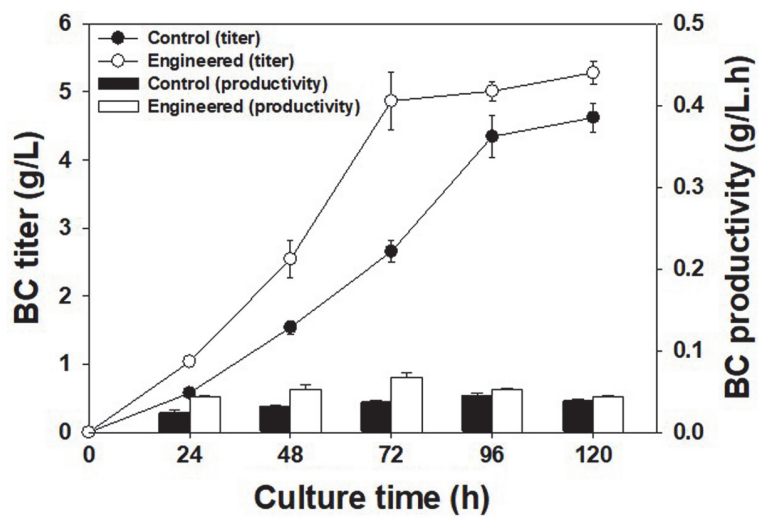

Fig. 4. BC production in engineered $K$. xylinus harboring pDHJCR15_pgm_galU_ndp. Control represents a wild-type K. xylinus. Symbols: Closed and open circles represent BC titers in control and engineered K. xylinus, respectively. Black and white bars represent $\mathrm{BC}$ productivity of control and engineered $K . x y l i n u s$, respectively. 
strain was $48.1 \mathrm{mg} / \mathrm{l} / \mathrm{h}$ at $96 \mathrm{~h}$ (Fig. 4). These results indicated that our engineered strain was capable of more efficient, faster, and higher production of BC.

\section{Conclusion}

In this study, we constructed a fully synthetic RBS library and successfully isolated novel synthetic RBSs with different strengths in K. xylinus cells using FACS screening. Using the synthetic RBSs, the expression levels of three genes ( $p g m$, gal $U$, and $n d p)$ involved in the biosynthesis of $\mathrm{BC}$ were optimized, and by reinforcing the metabolic pathway towards $\mathrm{BC}$ production, the enhanced production of $\mathrm{BC}$ could be achieved under both static and shaking culture conditions. To the best of our knowledge, this is the first study to engineer K. xylinus with synthetic parts to enhance the BC biosynthesis via high-throughput screening strategy. As we clearly demonstrated here (particularly in Fig. 3), the use of a stronger expression system does not always ensure higher production, although the balancing of expression level is more critical in multiple gene expressions to achieve higher production. For finer tuning of each gene expression, more synthetic parts including promoters, terminators, and regulators need to be further developed, and their use in the engineering of the BC biosynthesis pathway can serve to establish K. xylinus as a potential host for increased production of BC. Previously, we succeeded in engineering IS-elementresistant strain (K. xylinus SAIT-IS) by modifying a putative IS-element recognition sequence in a $b c s A$ gene, which resulted in 1.7-fold higher productivity [18]. We also believe that the introduction of the present optimized system (pDHJCR15_pgm_galU_ndp) with more synthetic parts in the engineered strain can provide a synergetic effect for BC production.

\section{Acknowledgment}

This work was supported by the Samsung Advanced Institute of Technology and by the Intelligent Synthetic Biology Center of the Global Frontier Project (Grant No. NRF-2014M3A6A8066443) funded by the Ministry of Science and ICT (MSIT).

\section{Conflict of Interest}

The authors have no financial conflicts of interest to declare.

\section{References}

1. Zhong C, Zhang GC, Liu M, Zheng XT, Han PP, Jia SR. 2013. Metabolic flux analysis of Gluconacetobacter xylinus for bacterial cellulose production. Appl. Microbiol. Biotechnol. 97: 6189-6199.

2. Mangayil R, Rajala S, Pammo A, Sarlin E, Luo J, Santala V, et al. 2017. Engineering and characterization of bacterial nanocellulose films as low cost and flexible sensor material. ACS Appl. Mater. Interfaces 9: 19048-19056.

3. Ha JH, Shah N, Ul-Islam M, Khan T, Park JK. 2011. Bacterial cellulose production from a single sugar a-linked glucuronic acid-based oligosaccharide. Process Biochem. 46: 1717-1723.

4. Sajadi E, Fatemi SSA, Babaeipour V, Deldar AA, Yakhchali B, Anvar MS. 2019. Increased cellulose production by heterologous expression of bcsA and B genes from Gluconacetobacter xylinus in E. coli Nissle 1917. Bioprocess Biosyst. Eng. 42: 2023-2034.

5. Buldum G, Bismarck A, Mantalaris A. 2018. Recombinant biosynthesis of bacterial cellulose in genetically modified Escherichia coli. Bioprocess Biosyst. Eng. 41:265-279.

6. Zhu H, Jia S, Wan T, Jia Y, Yang H, Li J, et al. 2011. Biosynthesis of spherical $\mathrm{Fe}_{3} \mathrm{O}_{4} /$ bacterial cellulose nanocomposites as adsorbents for heavy metal ions. Carbohydr. Polym. 86: 1558-1564.

7. Wu W, Tassi NG, Zhu H, Fang Z, Hu L. 2015. Nanocellulose-based translucent diffuser for optoelectronic device applications with dramatic improvement of light coupling. ACS Appl. Mater. Interfaces. 7: 26860-26864.

8. Jang WD, Kim TY, Kim HU, Shim WY, Ryu JY, Park JH, et al. 2019. Genomic and metabolic analysis of Komagataeibacter xylinus DSM 2325 producing bacterial cellulose nanofiber. Biotechnol. Bioeng. 116: 3372-3381.

9. Gwon H, Park K, Chung SC, Kim RH, Kang JK, Ji SM, et al. 2019. A safe and sustainable bacterial cellulose nanofiber separator for lithium rechargeable batteries. Proc. Natl. Acad. Sci. USA 116: 19288-19293.

10. Campano C, Balea A, Blanco A, Negro C. 2016. Enhancement of the fermentation process and properties of bacterial cellulose: a review. Cellulose 23: 57-91.

11. Zhang H, Ye C, Xu N, Chen C, Chen X, Yuan F, et al. 2017. Reconstruction of a genome-scale metabolic network of Komagataeibacter nataicola RZS01 for cellulose production. Sci. Rep. 7: 7911

12. Florea M, Hagemann H, Santosa G, Abbott J, Micklem CN, Spencer-Milnes X, et al. 2016. Engineering control of bacterial cellulose production using a genetic toolkit and a new cellulose-producing strain. Proc. Natl. Acad. Sci. USA 113: E3431-E3440.

13. Teh MY, Ooi KH, Danny Teo SX, Bin Mansoor ME, Shaun Lim WZ, Tan MH. 2019. An expanded synthetic biology toolkit for gene expression control in Acetobacteraceae. ACS Synth. Biol. 8: 708-723.

14. Yim SS, An SJ, Kang M, Lee J, Jeong KJ. 2013. Isolation of fully synthetic promoters for high-level gene expression in Corynebacterium glutamicum. Biotechnol. Bioeng. 110: 2959-2969.

15. Oh YH, Choi JW, Kim EY, Song BK, Jeong KJ, Park K, et al. 2015. Construction of synthetic promoter-based expression cassettes for the production of cadaverine in recombinant Corynebacterium glutamicum. Appl. Biochem. Biotechnol. 176: 2065-2075.

16. Gießelmann G, Dietrich D, Jungmann L, Kohlstedt M, Jeon EJ, Yim SS, et al. 2019. Metabolic engineering of Corynebacterium glutamicum for high-level ectoine production: design, combinatorial assembly, and implementation of a transcriptionally balanced heterologous ectoine pathway. Biotechnol. J. 14: 1800417.

17. Hestrin S, Schramm M. 1954. Synthesis of cellulose by Acetobacter xylinum. 2. Preparation of freeze-dried cells capable of polymerizing glucose to cellulose. Biochem. J. 58: 345-352.

18. Hur DH, Rhee HS, Lee JH, Shim WY, Kim TY, Lee SY, et al. 2020. Enhanced production of cellulose in Komagataeibacter xylinus by preventing insertion of IS element into cellulose synthesis gene. Biochem. Eng. J. 156: 107527.

19. Kim J, Yun J, Rhee H, Chung S. 2016. Vector replicable in E. coli and cell of genus Komagataeibacter, cell including the same, and method of using the same. U.S. Patent No. 9,976,150. Washington, DC: U.S. Patent and Trademark Office.

20. Seo SW, Yang JS, Kim I, Yang J, Min BE, Kim S, et al. 2013. Predictive design of mRNA translation initiation region to control prokaryotic translation efficiency. Metab. Eng. 15: 67-74.

21. Salis HM, Mirsky EA, Voigt CA. 2009. Automated design of synthetic ribosome binding sites to control protein expression. Nat. Biotechnol. 27: 946-950. 